\title{
Reduced DICER1 Expression Bestows Rheumatoid Arthritis Synoviocytes Proinflammatory Properties and Resistance to Apoptotic Stimuli
}

\author{
Ghada Alsaleh, ${ }^{1}$ Ramzi Nehmar, ${ }^{1}$ Stephan Blüml, ${ }^{2}$ Cédric Schleiss, ${ }^{1}$ Eleonore Ostermann, ${ }^{3}$ \\ Jean-Philippe Dillenseger, ${ }^{4}$ Amira Sayeh, ${ }^{5}$ Philippe Choquet, ${ }^{4}$ Doulaye Dembele, ${ }^{6}$ \\ Antoine Francois, ${ }^{3}$ Jean-Hugues Salmon, ${ }^{7}$ Nicodème Paul, ${ }^{1}$ Gernot Schabbauer, ${ }^{8}$ \\ Guillaume Bierry, ${ }^{9}$ Alain Meyer, ${ }^{10}$ Jacques-Eric Gottenberg, ${ }^{3}$ Gabrielle Haas, ${ }^{11}$ \\ Sebastien Pfeffer, ${ }^{11}$ Laurent Vallat, ${ }^{1} \mathrm{Jean} \mathrm{Sibilia}^{12}{ }^{\text {Seiamak Bahram, }}{ }^{1}$ and Philippe Georgel $^{1}$
}

Objective. While the regulatory role of individual microRNAs (miRNAs) in rheumatoid arthritis (RA) is well established, the role of $D I C E R 1$ in the pathogenesis of the disease has not yet been investigated. The purpose of this study was to analyze the expression of factors involved in miRNA biogenesis in fibroblast-like synoviocytes (FLS) from RA patients and to monitor the arthritis triggered by $\mathrm{K} / \mathrm{BxN}$ serum transfer in mice deficient in the Dicer gene (Dicer ${ }^{\mathrm{d} / \mathrm{d}}$ ).

Methods. The expression of genes and precursor miRNAs was quantified by quantitative reverse transcription-polymerase chain reaction (qRT-PCR).

Supported by the Agence Nationale de las Recherche (Laboratoire d'Excellence [LABEX] TRANSPLANEX; ANR-11-LABX0070 TRANSPLANTEX), INSERM, the University of Strasbourg, and the Institut Universitaire de France.

${ }^{1}$ Ghada Alsaleh, PharmD, PhD, Ramzi Nehmar, MS, Cédric Schleiss, MS, Nicodème Paul, PhD, Laurent Vallat, MD, PhD, Seiamak Bahram, MD, PhD, Philippe Georgel, PhD: INSERM UMRS1109, Fédération Hospitalo-Universitaire OMICARE, Centre de Recherche en Immunologie et Hématologie, and Université de Strasbourg, Strasbourg, France; ${ }^{2}$ Stephan Blüml, MD, PhD: Medical University of Vienna, Vienna, Austria; ${ }^{3}$ Eleonore Ostermann, $\mathrm{PhD}$, Antoine Francois, PhD, Jacques-Eric Gottenberg, $\mathrm{MD}, \mathrm{PhD}$ : INSERM UMR-S1109, Centre de Recherche en Immunologie et Hématologie, and Université de Strasbourg, Strasbourg, France, ${ }^{4}$ Jean-Philippe Dillenseger, MD, Philippe Choquet, PhD: Hôpitaux Universitaires de Strasbourg and CNRS, Université de Strasbourg, Strasbourg, France; ${ }^{5}$ Amira Sayeh, BS: CNRS, Université de Strasbourg, Strasbourg, France; ${ }^{6}$ Doulaye Dembele, PhD: Institut de Génétique et de Biologie Moléculaire et Cellulaire, INSERM U964 CNRS UMR-7104, and Université de Strasbourg, Illkirch, France; ${ }^{7}$ Jean-Hugues Salmon, MD: Centre Hospitalier Universitaire de Reims, Reims, France; ${ }^{8}$ Gernot Schabbauer, PhD: Institute for Physiology, Center for Physiology and Pharmacology, and Medical University of Vienna, Vienna, Austria; ${ }^{9}$ Guillaume Bierry, MD, PhD Hôpitaux Universitaires de Strasbourg and Université de Strasbourg, Strasbourg, France; ${ }^{10}$ Alain Meyer, MD: Hôpital de Hautepierre,
MicroRNA macroarray profiling was monitored by qRT-PCR. Cytokines were quantified by enzyme-linked immunosorbent assay. Experimental arthritis in mice was achieved by the transfer of serum from $\mathrm{K} / \mathrm{BxN}$ donors. Apoptosis was quantified using an enzymelinked immunosorbent assay.

Results. We found decreased DICERI and mature miRNA expression in synovial fibroblasts from RA patients. These cells were hyperresponsive to lipopolysaccharide, as evidenced by their increased interleukin-6 secretion upon stimulation. Experimental serum-transfer arthritis in Dicer ${ }^{\mathrm{d} / \mathrm{d}}$ mice confirmed that an unbalanced biogenesis of miRNAs correlated with an enhanced inflammatory response. Synoviocytes from both RA patients and Dicer ${ }^{\mathrm{d} / \mathrm{d}}$ mice exhibited increased resistance to apoptotic stimuli.

Conclusion. The findings of this study further substantiate the important role of DICER1 in the maintenance of homeostasis and the regulation of inflammatory responses.

Centre de Référence des Maladies Auto-immunes Rares, and Hôpitaux Universitaires de Strasbourg, Strasbourg, France; ${ }^{11}$ Gabrielle Haas, PhD, Sebastien Pfeffer, PhD: Architecture et Réactivité de l'ARN, UPR-9002, and Institut de Biologie Moléculaire et Cellulaire du CNRS, Strasbourg, France; ${ }^{12}$ Jean Sibilia, MD, PhD: Hôpital de Hautepierre, Centre de Référence des Maladies Auto-immunes Rares, Hôpitaux Universitaires de Strasbourg, and INSERM UMR-S1109, Fédération Hospitalo-Universitaire OMICARE, Centre de Recherche en Immunologie et Hématologie, and Université de Strasbourg, Strasbourg, France.

Address correspondence to Philippe Georgel, PhD, Centre de Recherche d'Immunologie et d'Hématologie, 1 Place de l'Hôpital, Strasbourg 67085, France. E-mail: pgeorgel@unistra.fr. Submitted for publication October 22, 2015; accepted in revised form February 9, 2016. 
The involvement of microRNAs (miRNAs) in the regulation of fundamental cellular mechanisms, such as development, differentiation, apoptosis, or cell division, is now supported by numerous findings indicating that dysfunction of miRNAs is associated with several human disorders (1). Among these is rheumatoid arthritis (RA), a multifactorial, complex disease of an immunoinflammatory nature that affects millions of people worldwide. In the last 5 years, several miRNAs (e.g., miR-146a [2], miR155 [3,4], miR-124a [5], and miR-203 [6]) that exhibit differential expression in samples from RA patients have been discovered (for review see refs. 7-9). Our group has contributed to the identification of miRNAs showing deregulated expression in activated synoviocytes: miR-346 $(10,11)$, miR-19 (12), and miR-20a $(13,14)$.

Surprisingly however, reports describing diseaseassociated global dysregulation of miRNA production are scarce. This appears to be at odds with the description of mutations in the DICER1 gene, which are associated with a diverse set of human diseases, mainly tumor-related, such as pleuropulmonary blastoma (15), cystic nephroma (16), nonepithelial ovarian cancer (17), multinodular goiter $(18,19)$, and Wilms' tumors $(20)$. In addition to DICER1, germline mutations in other components of the miRNA biosynthesis pathway (TARBP2, AGO2, TNRC6A, TNRC6C , and XPO5) are also associated with a predisposition to cancer in humans $(16,21,22)$. Therefore, an evaluation of the global impact of miRNAs on the inflammatory response might be relevant.

In the present study, we first investigated the expression of genes involved in the miRNA biogenesis machinery in synoviocytes from RA patients as compared to cells isolated from osteoarthritis (OA) patients and from healthy control subjects. We next evaluated the involvement of miRNAs in RA, upon global deregulation of the miRNA pathway in mice carrying a hypomorphic Dicer mutation (Dicer ${ }^{\mathrm{d} / \mathrm{d}}$ ), driving diminished expression of the gene. We also monitored the impact of reduced expression of DICER1 on lipopolysaccharide (LPS)dependent proinflammatory cytokine secretion and response to apoptotic stimuli in mouse mutant fibroblastlike synoviocytes (FLS) or in human FLS upon small interfering RNA (siRNA) DICER1-mediated knockdown.

\section{MATERIALS AND METHODS}

Patient characteristics. Human FLS were isolated from synovial tissues obtained from 15 RA patients, 15 OA patients, and 15 healthy subjects at the time of arthroscopic synovectomy of the knee joint (Supplementary Table 1, available on the Arthritis \& Rheumatology web site at http://onlinelibrary. wiley.com/doi/10.1002/art.39641/abstract). Human dermal fibroblasts were obtained by $4-\mathrm{mm}$ punch biopsy of the dorsal forearm of 4 patients with systemic sclerosis (Supplementary Table 1) and from the corresponding area of 3 healthy subjects. Systemic sclerosis patients had diffuse cutaneous disease and anti-Scl 70 antibodies. Minor salivary gland biopsy samples from 4 patients with primary Sjögren's syndrome (Supplementary Table 1) and 5 healthy controls were obtained for the primary culture of salivary gland epithelial cells. Samples were also obtained from 5 age- and sex-matched controls with sicca syndrome, but without any features of autoimmunity: no autoantibody; no salivary gland lymphocytic infiltrate. Informed consent was obtained from all donors.

Cell culture. Human FLS were isolated from synovial tissues obtained from RA and OA patients and healthy controls undergoing knee joint arthroscopic synovectomy. The diagnosis of RA conformed to the 2010 revised criteria of the American College of Rheumatology/European League Against Rheumatism (23).

FLS from mice were isolated from synovial tissues obtained from Dicer ${ }^{\mathrm{d} / \mathrm{d}}$ and littermate controls and were cultured as described previously (24). Experiments were performed between the third and ninth passages. Cell number and cell viability were checked by MTT assay. Human and mouse FLS $\left(2 \times 10^{5}\right.$ cells $)$ were stimulated with $1 \mathrm{ml}$ of medium alone or medium containing LPS $(1 \mu \mathrm{g} / \mathrm{ml})$.

Transfection assays. Transfection of human FLS with siRNA DICER (20 pM per sample) or with AllStars negative control siRNA was performed using a Human Dermal Fibroblast Nucleofector kit from Lonza (10). Human FLS were plated in 24 -well plates $\left(1 \times 10^{5}\right.$ cells/well $)$. At 48 hours after transfection, cells were stimulated with LPS $(1 \mu \mathrm{g} / \mathrm{ml})$ for 6 hours. Transfection efficiency was evaluated with the pmaxGFP vector. Total RNA was extracted using TRIzol, and the release of interleukin-6 (IL-6) was measured in culture supernatants by a heterologous 2 -site sandwich enzyme-linked immunosorbent assay (ELISA).

MTT and cell death assays. To evaluate the cell density, culture medium was removed and FLS $\left(1 \times 10^{4}\right.$ cells $)$ were resuspended at different time points $(24,48$, and 72 hours) in $200 \mu \mathrm{l}$ of a $0.4 \mathrm{mM}$ concentration of MTT dissolved in phosphate buffered saline and incubated for 4 hours at $37^{\circ} \mathrm{C}$. Staining solution was removed, and after cell lysis and formazan solubilization in $100 \mu \mathrm{l}$ of DMSO, optical density (OD) was measured at $570 \mathrm{~nm}$.

Cell death assay was performed on FLS $\left(1 \times 10^{4}\right.$ cells $)$ stimulated for 20 hours with $200 \mu \mathrm{l}$ of complete medium containing staurosporine $(1 \mu M)$ or FasL $(500 \mathrm{ng} / \mathrm{ml})$. Cell death was measured in cell lysate by ELISA (Cell Death Detection ELISA Plus; Roche Diagnostics). This method specifically detects mono- and oligonucleosomes (using monoclonal mouse antibodies directed against single-stranded and doublestranded DNA and histones H1, H2A, H2B, H3, and H4) in the cytoplasm of apoptotic cells, indicating apoptosisassociated DNA degradation. An enrichment factor was determined by calculating the OD of cells treated with an apoptosis-inducing agent (FasL or staurosporine) divided by the OD of the same samples before treatment.

Mice. Dicer-deficient mice (Dicer ${ }^{\mathrm{d} / \mathrm{d}}$ ) described previously (25) were backcrossed more than 10 times against C57BL/6 mice in our laboratory. Littermate controls are designated as Dicer ${ }^{+/+}$. Mice used in all experiments were age- and sex-matched. The animals were 
A

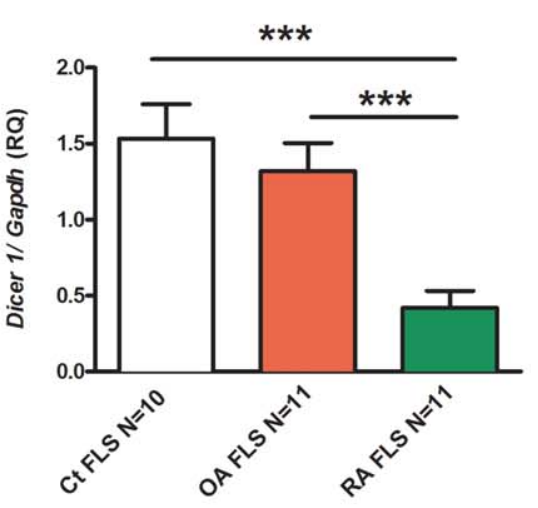

C

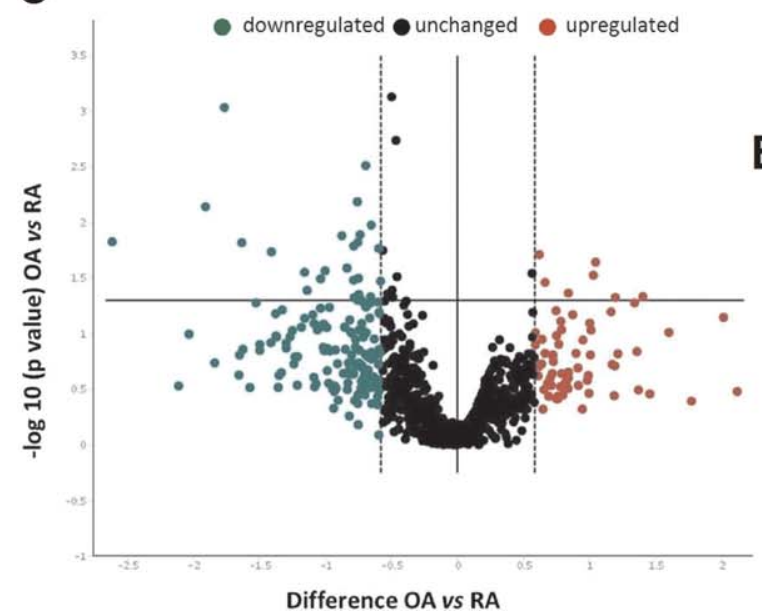

B

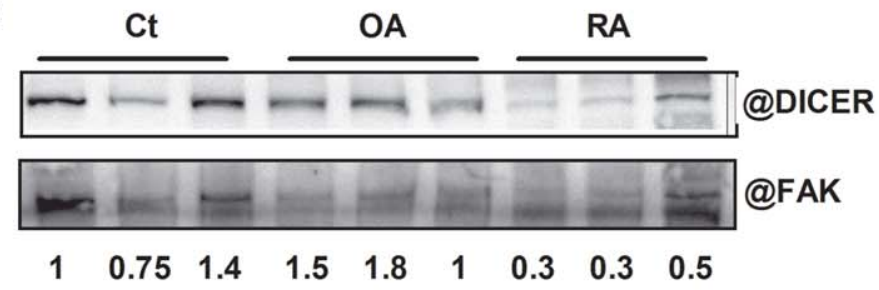

D

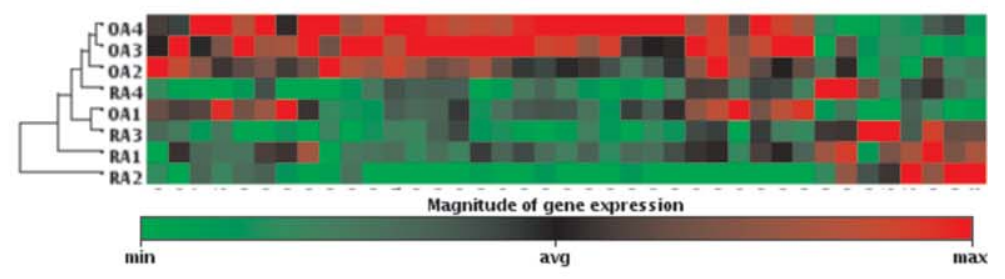

E

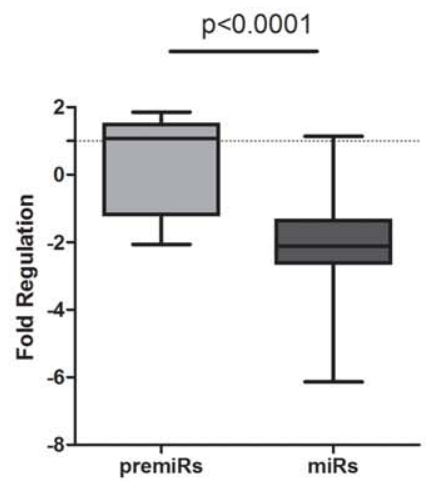

Figure 1. Diminished DICER and microRNA (miRNA) expression in rheumatoid arthritis (RA) fibroblast-like synoviocytes (FLS). A, DICER1 expression in primary FLS from biopsy samples obtained from healthy control (Ct) donors, osteoarthritis (OA) patients, and RA patients, as determined by quantitative reverse transcription-polymerase chain reaction (qRT-PCR). Results are normalized to GAPDH. Values are the mean \pm SEM. $* * *=P<0.0001$. B, Western blot showing DICER1 expression in FLS from biopsy samples obtained from 3 control subjects, 3 OA patients, and 3 RA patients. Expression of the focal adhesion kinase (FAK), a high molecular weight (125-kd) ubiquitously expressed protein, is shown as the loading control. Numbers indicate the intensity of the DICER1 signal normalized to that of FAK. C, Macroarray analysis of miRNAs isolated from RA $(n=4)$ and OA $(n=4)$ FLS. The volcano plot displays the relationship between the fold change and the significance between groups, using a scatterplot view. Expression of miRNAs below the detection level (threshold cycle $\left[\mathrm{C}_{\mathrm{t}}\right]<35$ ) was excluded from the analysis. The $\mathrm{x}$-axis represents the $\log _{2}$ fold change. Vertical dotted lines indicate a threshold of 1.5 -fold change in miRNA expression; horizontal line indicates the $P$ value of the $t$-test threshold $(P<0.05)$. D, Hierarchical cluster analysis of miRNA expression in 4 RA and 4 OA patients. Cluster analysis of the 39 miRNAs that were differentially expressed between OA and RA patients was done using online Qiagen software. E, Quantification of 20 precursor miRNAs (pre-miRs) or mature miRNAs (miRs) in FLS from 4 RA and 4 OA patients, as determined by qRT-PCR. Results are expressed as fold regulation $(-1 /$ fold change $[\mathrm{FC}])$, where OA patients represent the control group. Horizontal line represents a threshold of 1 (no difference between RA and OA samples). Data are shown as box plots. Each box represents the 25th to 75th percentiles. Lines inside the boxes represent the median. Lines outside the boxes represent the minimum and maximum of all the data. $P$ value was determined by Student's $t$-test. RQ= relative quantity; @ = antibody.

maintained under specific pathogen-free conditions in the Animal Care Facility of the Institut d'Immunologie et d'Hématologie in Strasbourg.

$\mathrm{K} / \mathrm{BxN}$ serum transfer-induced arthritis and clinical scoring. Serum was collected from 9-week-old $\mathrm{K} / \mathrm{BxN}$ mice. The samples were pooled and stored at $-80^{\circ} \mathrm{C}$ until used. Arthritis was induced in adult female Dicer ${ }^{\mathrm{d} / \mathrm{d}}$ and Dicer ${ }^{+/+}$ mice by 2 intraperitoneal injections of $\mathrm{K} / \mathrm{BxN}$ serum (150 $\mu \mathrm{l}$ each). In the "multiple flares" model, recipient mice were injected on days $0,1,22,23,42,43,63$, and 64. An articular arthritis index was determined by visual inspection and scoring of changes on a $0-4$ scale $(0=$ no swelling or erythema, $1=$ slight swelling and/or erythema, 2 = low-to-moderate edema, 3 = pronounced edema with limited use of the joint, and 4 = excessive edema with joint rigidity). Clinical indices for all 4 paws were added as a composite score. Hindpaw and forepaw thickness was measured with calipers.

Ethics information. Handling of mice and experimental procedures were conducted in accordance with the French Law for the Protection of Laboratory Animals. The 
procedures were approved by the Service Véterinaire de la Préfecture du Bas-Rhin (France; authorization no. A-67345) and by the Regional Ethical Committee for Animal Experimentation (CREMEAS) of Strasbourg University. Human cells were obtained after informed consent was given by the donors.

Data sharing. Data reported herein (including the supplementary data) will be shared if needed. The miRNA macroarray data are available at the NCBI Gene Expression Omnibus (GEO) database (accession no. GSE72564).

Statistical analysis. The nonparametric MannWhitney unpaired 2-tailed $t$-test was used to compare 2 independent groups. Pearson's correlation was performed to analyze the relationship between different parameters, and linear regression was used to monitor progression over time by comparing the slopes. In all cases, we used GraphPad 5.04 software. $P$ values less than 0.05 were considered significant.

\section{RESULTS}

Decreased DICERI expression in RA FLS. To obtain an overview of the miRNA expression landscape in RA, we investigated DICER1 transcripts by quantitative reverse transcription-polymerase chain reaction (qRT-PCR) in FLS from patients with RA. Primary cells cultured from biopsy samples obtained from healthy controls or from OA patients were used as controls. In this setting, we observed significant reduction of DICER1 mRNAs $(P<0.001)$ in FLS from RA patients $(\mathrm{n}=11)$ (see Supplementary Table 1$)$ as compared to healthy controls $(\mathrm{n}=10)$ or OA patients $(\mathrm{n}=11)$ (Figure 1A). This differential DICER1 expression was not observed in peripheral blood mononuclear cells from RA patients $(\mathrm{n}=10)$ as compared to healthy donors $(\mathrm{n}=10)$ (data not shown). Western blotting confirmed decreased DICER1 expression in RA FLS as compared to OA or healthy FLS (Figure 1B).

To demonstrate the biologic significance of lower expression of DICER1 in RA FLS, we used low-density arrays to analyze 1,066 miRNAs (miRBase V16) by qRT-PCR in cultured FLS from 4 RA and 4 OA patients (normalized data and analyses shown in Supplementary Table 2, available at http://onlinelibrary. wiley.com/doi/10.1002/art.39641/abstract). Raw data have been deposited in the NCBI Gene Expression Omnibus (GEO) database (GSE72564). This experiment revealed that the overall fold regulation was significantly below the theoretical value of $1(P<0.0001)$. Thirty-nine miRNAs exhibited significant differential expression between the two conditions $(P<0.05), 80 \%$ of which showed reduced expression in RA FLS. This is illustrated in Figure 1C. Notably, unsupervised hierarchical clustering of these 39 miRNAs enabled the segregation of RA versus OA patients, with the exception of sample OA1, which was characterized by down-regulated genes similar to those seen in the RA samples (Figure 1D). This phenotype may be accounted for by the fact that sample OA1 was from the oldest OA patient.

DICER1-specific involvement was demonstrated by using qRT-PCR to analyze the expression of 20 mature miRNAs (miR-19b, 199b, 577, let-7c, 10b, 1260a, 328, 34b, 3182, 670, 2116, 190a, 597, 497, 610, $30 \mathrm{c}-2,502,585,218-1,125 \mathrm{~b}-2$, and 1246) and the corresponding precursor microRNAs (pre-miRs) in FLS from 4 RA and 4 OA samples. As shown in Figure 1E, the fold regulation of mature miRNAs was significantly decreased $(P<0.0001)$ compared to that of pre-miRs, which illustrates lower expression of miRNAs in RA samples than in OA.

Next, we quantified DICER1 transcripts in similar cell types involved in 2 other inflammatory diseases: systemic sclerosis and Sjögren's syndrome. Human dermal fibroblasts were cultured from skin biopsy samples obtained from patients with systemic sclerosis or from healthy controls, and salivary gland epithelial cells were obtained from patients with primary Sjögren's syndrome or from controls with sicca symptoms but without any features of an autoimmune disease (Supplementary Table 1). Similar levels of expression were observed in the different samples (Figure 2A), irrespective of origin. We also quantified the expression of all genes involved in the miRNA biosynthesis machinery in RA FLS (Figure 2B) and found that these cells specifically exhibited a defect in DICER1 expression. Taken together, these data reveal reduced DICER1 expression as a specific feature of RA FLS.

Worsened joint inflammation in $\operatorname{Dicer}^{\mathrm{d} / \mathrm{d}}$ mice upon injection of arthritogenic serum. To investigate the consequences of reduced Dicer expression in an animal model, we used a mouse line with a hypomorphic mutation in the Dicer gene $\left(\right.$ Dicer $\left.^{\mathrm{d} / \mathrm{d}}\right)$, in which low levels of Dicer expression still enables the production of viable adults $(25,26)$. We first confirmed that Dicer transcripts were reduced in FLS from the Dicer ${ }^{\mathrm{d} / \mathrm{d}}$ mice (Figure 3A), whereas the expression of the other genes of the miRNA biogenesis pathway remained unaffected, a situation comparable to that in human RA FLS (Figure 2B). The Dicer ${ }^{\mathrm{d} / \mathrm{d}}$ mutation affects a subset of miRNAs (26), and our qRT-PCR analysis of selected miRNAs homologous to those associated with RA in humans reproduced this trend. As shown in Figure 3B, the amount of several (9 of 17) miRNAs was significantly reduced in FLS from Dicer ${ }^{\mathrm{d} / \mathrm{d}}$ mice. Notably, despite this significant reduction in miRNA expression, Dicer ${ }^{\mathrm{d} / \mathrm{d}}$ mutant adult mice appeared normal and did not spontaneously develop any overt inflammatory phenotype. 
A
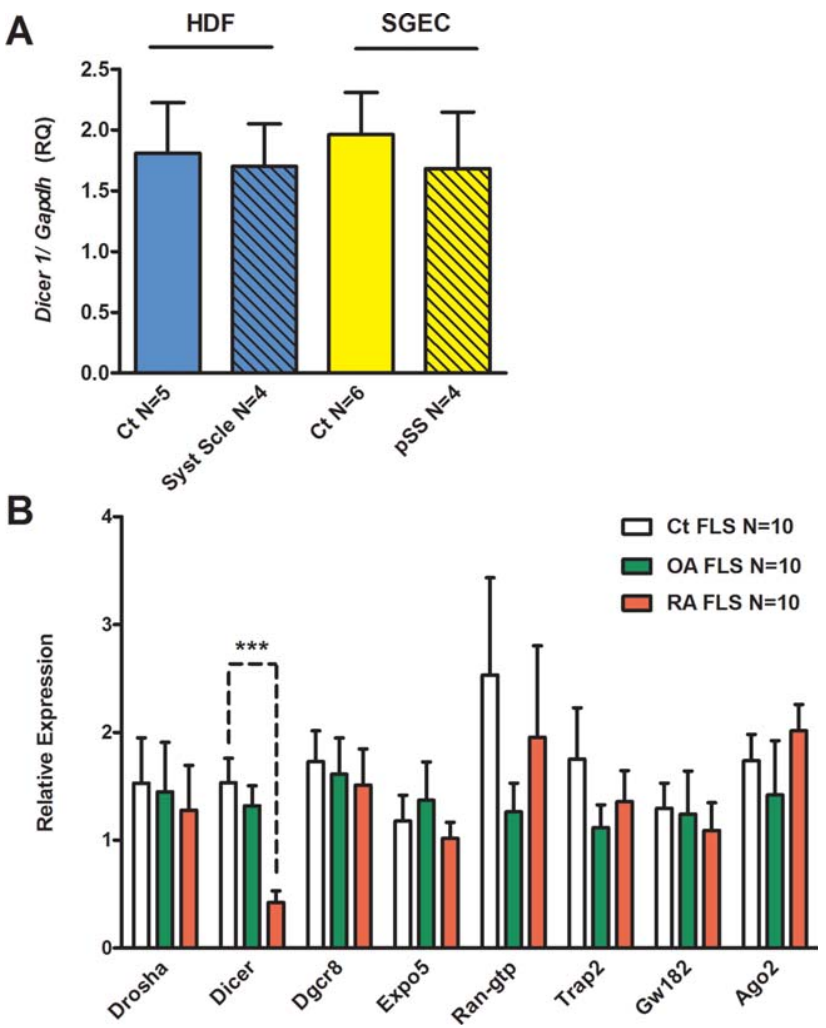

Figure 2. Specific down-modulation of DICER1 expression in RA FLS. A, DICER1 expression in human dermal fibroblasts (HDFs) from skin biopsy samples obtained from healthy control donors and systemic sclerosis (Syst Scle) patients, as well as in salivary gland epithelial cells (SGECs) from healthy control donors and primary Sjögren's syndrome (pSS) patients, as determined by qRT-PCR. Results are normalized to GAPDH. B, Quantification of all of the genes participating in the miRNA biogenesis pathway in FLS from healthy control donors, OA patients, and RA patients. Results are expressed relative to GAPDH. Values are the mean \pm SEM. $* * *=P<0.0001$. See Figure 1 for other definitions. Color figure can be viewed in the online issue, which is available at http://onlinelibrary. wiley.com/journal/doi/10.1002/art.39641/abstract.

In order to test the impact of limited Dicer expression, we induced arthritis by injecting the animals with $\mathrm{K} / \mathrm{BxN}$ mouse sera (27). We observed a significant increase in joint inflammation in Dicer ${ }^{\mathrm{d} / \mathrm{d}}$ mice $8-12$ days following serum transfer, and increased swelling of the soft tissues of Dicer ${ }^{\mathrm{d} / \mathrm{d}}$ mice was noted by use of micro-computed tomography on day 9 (Supplementary Figure 1, available at http://onlinelibrary.wiley.com/doi/ 10.1002/art.39641/abstract).

We then reproduced in Dicer ${ }^{\mathrm{d} / \mathrm{d}}$ mice successive flares of arthritis, a characteristic feature of RA in humans, by 4 successive injections of serum. The evolution of the arthritis score over 88 days is shown in Figure 4A. At the peak of the first flare, Dicer ${ }^{\mathrm{d} / \mathrm{d}}$ mice exhibited more severe symptoms, a phenomenon that was repeatedly observed during the next 3 waves. In addition, repeated injections of $\mathrm{K} / \mathrm{BxN}$ serum every 21-22 days also indicated that Dicer ${ }^{\mathrm{d} / \mathrm{d}}$ mice failed to recover from the induced inflammation. Linear regression analysis of the scores measured on days $21,42,63$, and 84 in wild-type $\mathrm{Dicer}^{+/+}$ mice and mutant Dicer ${ }^{\mathrm{d} / \mathrm{d}}$ mice (Figure 4B) indicated that the slope of the 2 curves was significantly different $(P<0.001)$. This clearly demonstrates that from day 42 to the end of the experiment on day 88 , Dicer ${ }^{\mathrm{d} / \mathrm{d}}$ mice exhibited worsening of their arthritic symptoms. This was corroborated by a similar analysis performed on the hindpaw and forepaw thickness measurements in the same mice (Figures 4C and D). Additionally, after the second flare, we noticed severe swelling of the toes in the Dicer ${ }^{\mathrm{d} / \mathrm{d}}$
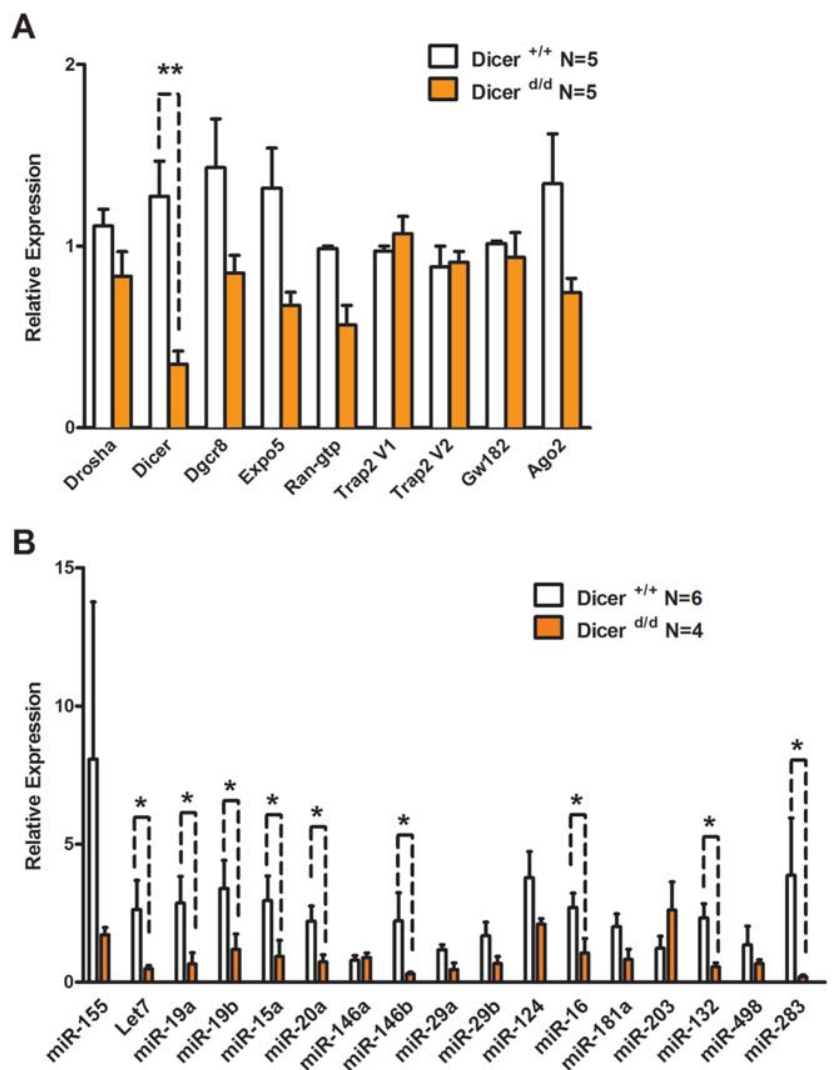

Figure 3. Decreased miRNA maturation in Dicer-deficient (Dicer ${ }^{\mathrm{d} / \mathrm{d}}$ ) mice. A, Expression of the genes involved in the miRNA machinery in FLS from Dicer littermate control $\left(\right.$ Dicer $\left.^{+/+}\right)$mice and Dicer-mutant $\left(\right.$ Dicer $\left.^{\mathrm{d} / \mathrm{d}}\right)$ mice, as determined by qRT-PCR. B, Expression of individual miRNAs in FLS from Dicer ${ }^{+/+}$littermate control mice and Dicer ${ }^{\mathrm{d} / \mathrm{d}}$ mice, as determined by qRT-PCR. Results are expressed relative to GAPDH in A and relative to U6 RNA in B. The data are representative of 3 independent experiments using similar numbers of mice. Values are the mean \pm SEM. $*=P<0.05 ; * *=P<0.001$. See Figure 1 for other definitions. Color figure can be viewed in the online issue, which is available at http://onlinelibrary.wiley.com/journal/doi/10.1002/art. 39641/abstract. 


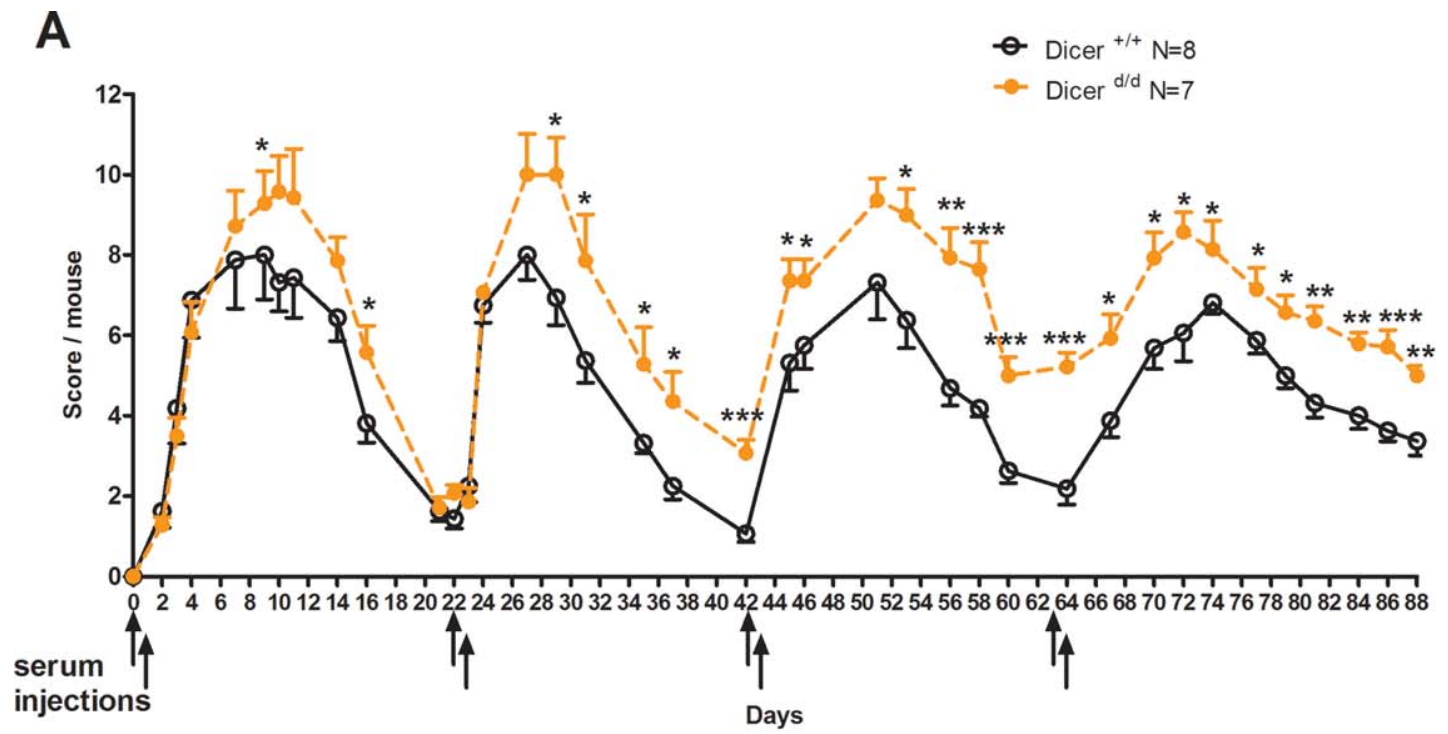

B

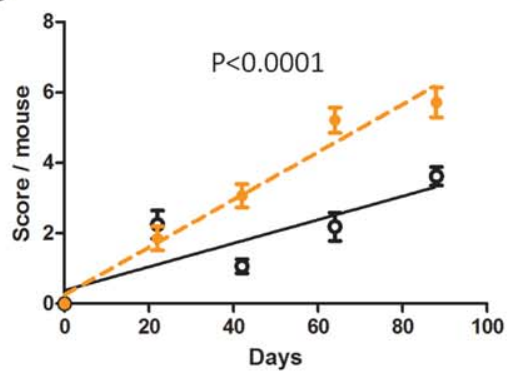

C

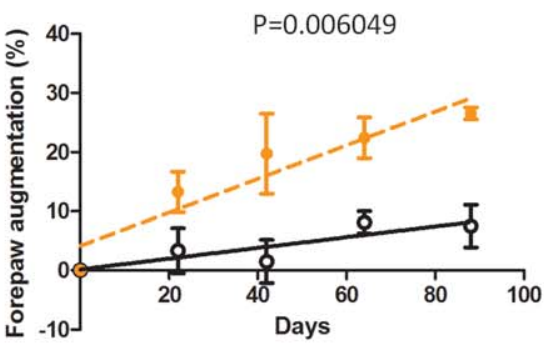

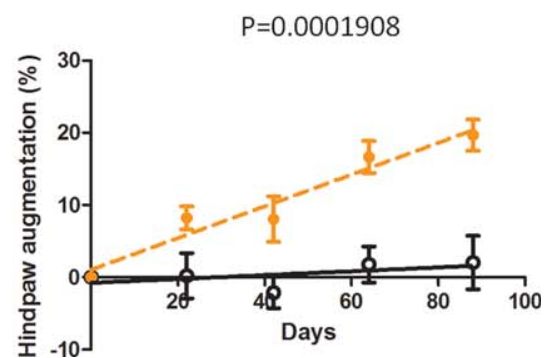

Figure 4. Worsening of rheumatoid arthritis symptoms in Dicer-deficient $\left(\right.$ Dicer $\left.^{\mathrm{d} / \mathrm{d}}\right)$ mice upon successive injections of $\mathrm{K} / \mathrm{BxN}$ mouse serum. A, Evolution of arthritis scores over 88 days in Dicer littermate control $\left(\operatorname{Dicer}^{+/+}\right)$mice and Dicer-mutant $\left(\operatorname{Dicer}^{\mathrm{d} / \mathrm{d}}\right)$ mice. Arrows indicate injections of $\mathrm{K} / \mathrm{BxN}$ mouse serum $(150 \mu \mathrm{l}$; days $0,1,22,23,42,43,63$, and 64). B-D, Linear regression analysis of the scores obtained on days 22, 42, 63, and 88 (B), as well as of the thickness of the forepaws $(\mathbf{C})$ and hindpaws $(\mathbf{D})$, in Dicer ${ }^{+/+}$littermate control mice and Dicer ${ }^{\mathrm{d} / \mathrm{d}}$ mice. Results are representative of 2 experiments with similar numbers of mice. Values are the mean \pm SEM. $*=P<0.05 ; * *=P<0.001 ; * * *=P<0.0001$. Color figure can be viewed in the online issue, which is available at http://onlinelibrary.wiley.com/journal/doi/10.1002/art.39641/abstract.

mice (Supplementary Figures 2A and B, available at http:// onlinelibrary.wiley.com/doi/10.1002/art.39641/abstract).

Histologic analysis of the joints harvested from these animals on day 88 was performed. We recorded the mean area of the synovial membrane inflammation, the mean area of the tarsal zone erosion, and the number of osteoclasts in this area. Taken individually, none of these parameters enabled significant discrimination between the Dicer ${ }^{\mathrm{d} / \mathrm{d}}$ mice and the control group, albeit each appeared to be increased in the mutant mice. Correlation analyses (see Supplementary Figures 3A-C, available at http://onlinelibrary.wiley.com/doi/10.1002/ art.39641/abstract) showed, as expected, an association between inflammation and erosion and between erosion and the amount of osteoclasts in both wild-type and mutant animals. Interestingly, we noted that the amount of osteoclasts also correlated positively with synovial inflammation only in the mutant mice.
Aberrant inflammatory and apoptotic responses in FLS from Dicer/d mice. To analyze the functional effects of low DICER1 expression in human FLS, we quantified IL-6 expression and secretion in synoviocytes cultured from RA and OA patients as well as from healthy donors (Figure 5A). IL-6 is a major proinflammatory cytokine in the pathogenesis of RA, and interestingly, its basal expression appeared augmented in primary RA FLS at the transcription and protein levels. This difference was also observed in LPS-activated FLS. Because RA FLS also exhibit down-modulation of DICER1 expression, we tested whether transfection of siRNA directed against DICER1 would either mimic this phenotype in healthy cells or even amplify it in RA FLS. We observed increased IL6 transcription upon DICER1 knockdown in FLS isolated from control subjects, OA patients, and RA patients under steady-state conditions (Figure 5B). However, when LPS was used 
A
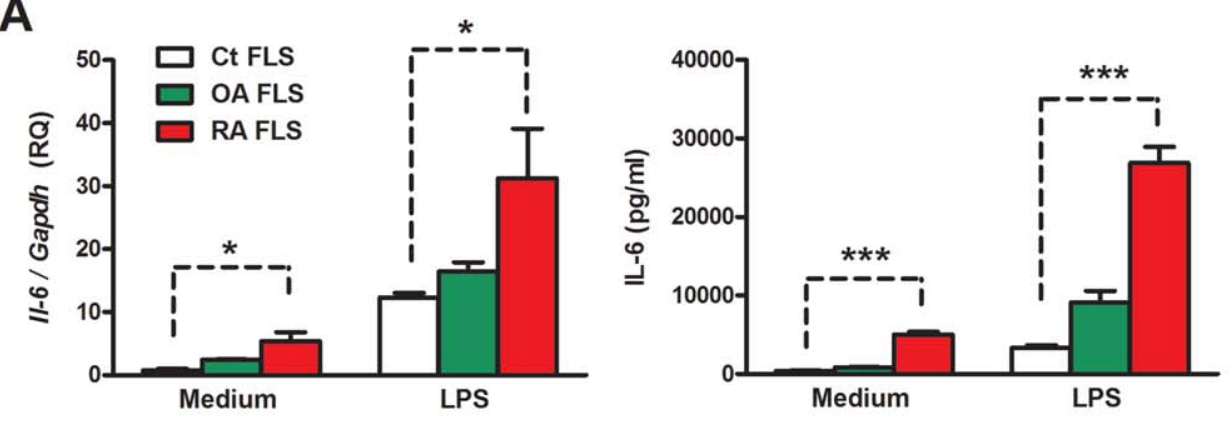

B
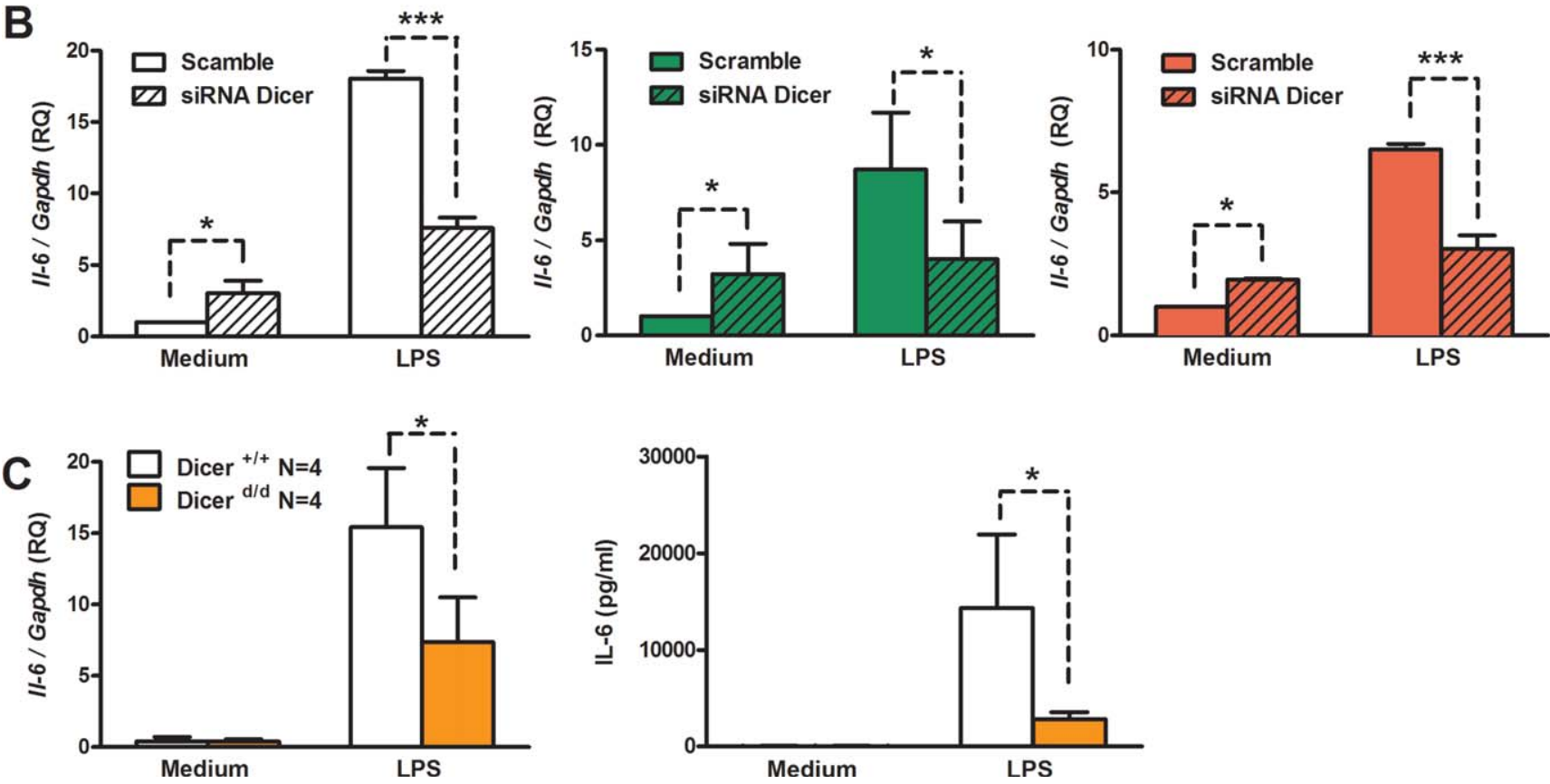

Figure 5. Functional impact of reduced expression of DICER1 on the expression of interleukin-6 (IL-6). A, Quantification of IL-6 in FLS from healthy control donors, OA patients, and RA patients $(\mathrm{n}=3$ per group). Cells were left untreated (medium) or were treated for 6 hours with lipopolysaccharide (LPS), and then IL6 transcripts were quantified by qRT-PCR (normalized to GAPDH) (left), and IL-6 secretion in the supernatant was measured by enzyme-linked immunosorbent assay (ELISA) (right). B, Quantification of IL6 transcripts in FLS from healthy control donors (left), OA patients (middle), and RA patients (right) following transfection with irrelevant small interfering RNA (siRNA) (scramble) or with DICER1 siRNA, as determined by qRT-PCR. Cells were transfected for 48 hours and then activated with LPS for 6 hours. C, Quantification of IL-6 transcripts in FLS from Dicer littermate control $\left(\operatorname{Dicer}^{+/+}\right)$mice and Dicer-mutant $\left(\mathrm{Dicer}^{\mathrm{d} / \mathrm{d}}\right)$ mice. Cells were left untreated or were treated for 6 hours with LPS, and then Il6 transcripts were quantified by qRT-PCR (left), and IL-6 secretion in the supernatant was measured by ELISA (right). Results are representative of 3 independent experiments with similar numbers of mice. Values are the mean \pm SEM. $*=P<0.05 ; * * *=P<0.0001$. See Figure 1 for other definitions. Color figure can be viewed in the online issue, which is available at http://onlinelibrary.wiley.com/journal/doi/10.1002/art.39641/abstract.

to activate these cells, a significantly lower number of IL-6 transcripts were detected when DICER1 expression was knocked-down upon siRNA transfection. This is in sharp contrast with our previous observation indicating that IL-6 is expressed at a higher level in RA FLS and indicates that the sole impairment of DICER1 does not recapitulate the genetic complexity which accounts for the defects manifested in RA synoviocytes. Nevertheless, this experiment indicated that DICER1 is likely an important player in cytokine homeostasis.
We also examined the inflammatory response of wild-type and Dicer ${ }^{\mathrm{d} / \mathrm{d}}$ murine FLS (Figure 5C), which confirmed that LPS-dependent expression of IL-6 is decreased at both the RNA and protein levels in cells harvested from mutant mice. This result might seem to conflict with our in vivo results showing increased inflammation in Dicer ${ }^{\mathrm{d} / \mathrm{d}}$ mice upon $\mathrm{K} / \mathrm{BxN}$ serum transfer. However, this apparent discrepancy must be interpreted in view of the mechanisms, mostly complement-dependent and IL-6 independent (28), that are responsible for the inflammatory response that is trig- 
gered upon serum transfer. The inflammatory milieu itself could account for the reduced DICER1 expression (29). To explore this possibility, we quantified DICER1 expression in FLS isolated from healthy donors and stimulated with various inflammatory cytokines. We observed that addition of IL-6 to the culture medium of FLS can induce such down-modulation of DICER1 expression (Supplementary Figure 4, available at http://onlinelibrary.wiley. com/doi/10.1002/art.39641/abstract). However, Dicer expression in cultured FLS isolated from $\mathrm{K} / \mathrm{BxN}$ animals, which exhibit high levels of IL-6 in the joints (28), remains comparable to that of controls (Supplementary Figure 5, available at http://onlinelibrary.wiley.com/doi/ 10.1002/art.39641/abstract), incidentally reflecting the gap between the animal model and the human disease.

We observed that cultured Dicer ${ }^{\mathrm{d} / \mathrm{d}}$ FLS systematically reached confluence faster than control cells did (reflecting augmented cell number, as judged by MTT staining) (Supplementary Figure 6, available at http://onlinelibrary.wiley.com/doi/10.1002/art.39641/abstract), indicative of increased proliferation and/or diminished apoptosis. Whereas proliferation assays quantifying 5-bromo- $2^{\prime}$ deoxyuridine incorporation did not show differences between FLS from wild-type Dicer ${ }^{+/+}$and Dicer ${ }^{\mathrm{d} / \mathrm{d}}$ mice under normal culture conditions or upon LPS stimulation (data not shown), we noted that cells from Dicer ${ }^{\mathrm{d} / \mathrm{d}}$ mice were resistant to FasL- or staurosporine-induced apoptosis (Figures 6A and B). Interestingly, FLS from the RA patients exhibited similar behavior (Figure 6C).

\section{DISCUSSION}

RA is a multifactorial disease involving more than 100 risk loci $(30,31)$. In addition to these proteincoding genes, several miRNAs exhibiting deregulated expression have also been associated with disease susceptibility (32), and single-nucleotide polymorphisms close to some of these miRNAs have been linked to the occurrence of RA $(33,34)$. Our present analysis revealed significantly reduced DICER1 expression in FLS isolated from RA patients.

The mechanism by which reduced DICERI expression correlates with enhanced RA pathogenesis remains to be resolved, but 2 possibilities emerge from our finding of a positive correlation between the quantity of osteoclasts and synovial inflammation (Supplementary Figure 3C) in Dicer ${ }^{\mathrm{d} / \mathrm{d}}$ mice. In this context, augmented osteoclastogenesis triggered by low miRNA production may participate in the maintenance of an inflammatory milieu, or alternatively, excessive inflammation driven by loss of miRNA-dependent negative regulation sends a positive signal to promote osteoclas-
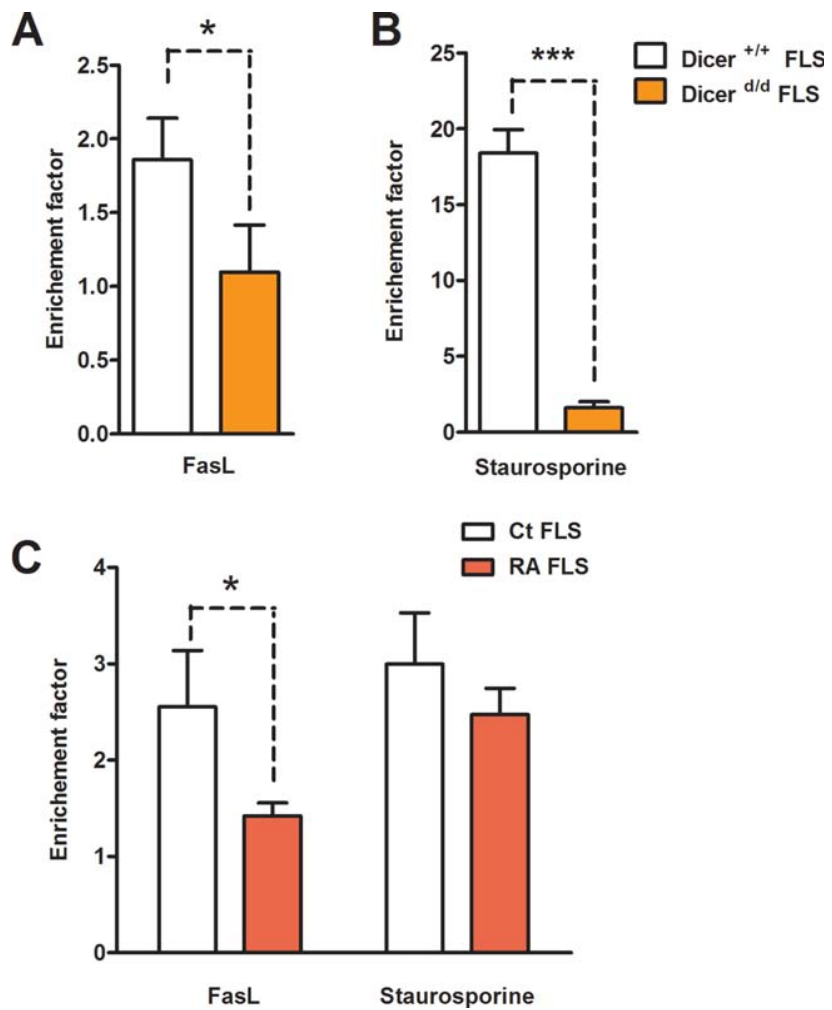

Figure 6. Increased resistance to apoptotic stimulation in FLS from Dicer-deficient $\left(\right.$ Dicer $\left.^{\mathrm{d} / \mathrm{d}}\right)$ mice. $\mathbf{A}$ and $\mathbf{B}$, Measurement of apoptosis of FLS from Dicer littermate control $\left(\right.$ Dicer $\left.^{+/+}\right)$mice and Dicer ${ }^{\mathrm{d} / \mathrm{d}}$ mice in response to FasL (A) and staurosporine $(\mathbf{B})(n=4$ mice per group). Cells were incubated with $500 \mathrm{ng} / \mathrm{ml}$ of FasL or with $1 \mu M$ staurosporine, and cell death was determined as mononucleosome quantification by enzyme-linked immunosorbent assay. The enrichment factor corresponds to the ratio of the optical density in samples treated with apoptosis-inducing agents to that of the same samples before treatment. Results are representative of 2 independent experiments. C, Measurement of apoptosis of FLS from healthy control donors and RA patients in response to FasL and staurosporine $(\mathrm{n}=5$ per group). Cells were stimulated and apoptosis was determined as in $\mathbf{A}$ and B. $*=P<0.05 ; * * *=P<0.0001$. See Figure 1 for other definitions. Color figure can be viewed in the online issue, which is available at http://onlinelibrary.wiley.com/journal/doi/10. 1002/art.39641/abstract.

togenesis. Ex vivo analysis of IL-6 expression showed that primary RA FLS expressed increased amounts of this cytokine (Figure 5A), similar to healthy FLS in which DICER1 had been knocked down upon siRNA transfection (Figure 5B). This suggests that miRNAs, which are important regulators for avoiding the spontaneous overexpression of potentially harmful mediators of inflammation, could indirectly affect the differentiation of osteoclasts, a process which was recently shown to be cytokine-dependent (35), and supports the second model. However, preliminary data from our laboratory indicate that low miRNA conditions favor osteoclast 
formation ex vivo, which supports the first hypothesis and the alternative concept recently formulated (36) and suggests that bone loss may precede inflammation.

Alternatively, miRNA-independent roles of DICER1 should also be considered. Indeed, DICER1 is engaged in complexes carrying various functions and is involved in multiple non-miRNA-related processes, such as chromatin assembly or nuclear receptor signaling (37). Of note, DICER1-dependent processing of Alu-encoded RNAs is a major player in avoiding the accumulation of large amounts of double-stranded RNA in retinal pigmented epithelial cells and thereby protects them from NLRP3-dependent excessive inflammation $(38,39)$. Considering the modest reduction in mature miRNA production observed in RA FLS, it is conceivable that this mechanism could also be at play in synoviocytes.

Notable information derived from our animal model is the absence of spontaneous RA symptoms in Dicer ${ }^{\mathrm{d} / \mathrm{d}}$ mice before serum injection, thus demonstrating that Dicer deficiency per se does not trigger arthritis. Furthermore, LPS stimulation of cells in which DICER1 has been knocked down (or of FLS isolated from Dicer ${ }^{\mathrm{d} / \mathrm{d}}$ mice) led to diminished IL-6 expression, contrary to the results in RA FLS, which showed increased IL-6 production. Hence, low levels of DICER activity in mice do not mimic the complex genetic and environmental networks that, when deregulated, lead to RA in humans. However, we performed repeated injections of serum, thereby creating "multiple flares" of arthritis, with the aim of reproducing the successive episodes of RA flares that generally occur in patients. These experiments showed that reduced expression of Dicer enhanced the RA-like symptoms at every peak following serum transfer. Importantly, diminished Dicer expression in the Dicer ${ }^{\mathrm{d} / \mathrm{d}}$ mice may create the appropriate conditions for the establishment of chronic joint inflammation and tissue damage. Interestingly, the reduction of miRNA expression rarely leads to dramatic phenotypes, unless aggravated by stress (40), which precisely corresponds to our observations in the Dicer ${ }^{\mathrm{d} / \mathrm{d}}$ mutant mice.

Notwithstanding that the KRN joint inflammation, which is essentially complement-driven, is quite distantly related to the human disease, which is mostly tumor necrosis factor-dependent, our adaptation of this model generated mice with chronic inflammation that showed tissue destruction in the joints and appeared to be more clinically relevant.

Patients with autoimmune diseases such as RA have a higher risk of developing certain lymphomas $(41,42)$. The precise links between these 2 diseases are essentially unknown, but both share some similar features, among which proliferation appears to be crucial. Thus, mutations in several cancer genes, such as $P 53$, which is involved in the control of cell proliferation, have already been identified as risk susceptibility genes in RA (43). Up to now, DICER1 variants have been described in diseases that are caused by cell proliferation defects (ovarian cancer, pleuropulmonary blastoma, hyperplasia of the thyroid gland parenchyma in goiter). Interestingly, our own analysis of FLS from patients with RA or FLS from Dicer ${ }^{\mathrm{d} / \mathrm{d}}$ mice showed that these cells are highly resistant to FasL- or staurosporine-induced apoptosis as compared to controls (Figure 6), a feature that may account for the synovial hyperplasia observed in RA patients.

More functional insights will be obtained upon analysis of a larger cohort of patients and correlation analyses between DICER1 expression and various parameters of RA, such as the Disease Activity Score in 28 joints and the C-reactive protein level. Nevertheless, our work already brings DICER1 to the core of interplay between inflammation, stress, and proliferation, all major contributors to the pathogenesis of RA.

\section{ACKNOWLEDGMENTS}

We thank Prof. François Bonnomet (Department of Orthopedic Surgery) for synovial biopsy samples, Angélique Pichot and Cécile Macquin for technical help, and Bernhard Ryffel (UMR-7355 CNRS, University of Orléans, Orléans, France) and Kim Midwood (The Kennedy Institute of Rheumatology, University of Oxford, Oxford, UK) for critical reading of the manuscript.

\section{AUTHOR CONTRIBUTIONS}

All authors were involved in drafting the article or revising it critically for important intellectual content, and all authors approved the final version to be published. Dr. Georgel had full access to all of the data in the study and takes responsibility for the integrity of the data and the accuracy of the data analysis.

Study conception and design. Alsaleh, Nehmar, Ostermann, Georgel. Acquisition of data. Alsaleh, Nehmar, Blüml, Schleiss, Sayeh, Francois, Haas, Georgel.

Analysis and interpretation of data. Alsaleh, Nehmar, Blüml, Dillenseger, Choquet, Dembele, Francois, Salmon, Paul, Schabbauer, Bierry, Meyer, Gottenberg, Pfeffer, Vallat, Sibilia, Bahram, Georgel.

\section{REFERENCES}

1. Mendell JT, Olson EN. MicroRNAs in stress signaling and human disease. Cell 2012;148:1172-87.

2. Nakasa T, Miyaki S, Okubo A, Hashimoto M, Nishida K, Ochi M, et al. Expression of microRNA-146 in rheumatoid arthritis synovial tissue. Arthritis Rheum 2008;58:1284-92.

3. Blüml S, Bonelli M, Niederreiter B, Puchner A, Mayr G, Hayer S, et al. Essential role of microRNA-155 in the pathogenesis of autoimmune arthritis in mice. Arthritis Rheum 2011;63:1281-8.

4. Kurowska-Stolarska M, Alivernini S, Ballantine LE, Asquith DL, Millar NL, Gilchrist DS, et al. MicroRNA-155 as a proinflammatory regulator in clinical and experimental arthritis. Proc Natl Acad Sci U S A 2011;108:11193-8. 
5. Nakamachi Y, Kawano S, Takenokuchi M, Nishimura K, Sakai Y, Chin T, et al. MicroRNA-124a is a key regulator of proliferation and monocyte chemoattractant protein 1 secretion in fibroblast-like synoviocytes from patients with rheumatoid arthritis. Arthritis Rheum 2009;60:1294-304.

6. Stanczyk J, Ospelt C, Karouzakis E, Filer A, Raza K, Kolling C, et al. Altered expression of microRNA-203 in rheumatoid arthritis synovial fibroblasts and its role in fibroblast activation. Arthritis Rheum 2011;63:373-81.

7. Wittmann J, Jack HM. MicroRNAs in rheumatoid arthritis: midget RNAs with a giant impact. Ann Rheum Dis 2011;70 Suppl 1:i92-6.

8. Tomankova T, Petrek M, Gallo J, Kriegova E. MicroRNAs: emerging regulators of immune-mediated diseases. Scand $\mathrm{J}$ Immunol 2012;75:129-41.

9. Ceribelli A, Nahid MA, Satoh M, Chan EK. MicroRNAs in rheumatoid arthritis. FEBS Lett 2011;585:3667-74.

10. Alsaleh G, Suffert G, Semaan N, Juncker T, Frenzel L, Gottenberg JE, et al. Bruton's tyrosine kinase is involved in miR-346related regulation of IL-18 release by lipopolysaccharideactivated rheumatoid fibroblast-like synoviocytes. J Immunol 2009;182:5088-97.

11. Semaan N, Frenzel L, Alsaleh G, Suffert G, Gottenberg JE, Sibilia J, et al. miR-346 controls release of TNF- $\alpha$ protein and stability of its mRNA in rheumatoid arthritis via tristetraprolin stabilization. PloS One 2011;6:e19827.

12. Philippe L, Alsaleh G, Suffert G, Meyer A, Georgel P, Sibilia J, et al. TLR2 expression is regulated by microRNA miR-19 in rheumatoid fibroblast-like synoviocytes. J Immunol 2012;188:454-61.

13. Philippe L, Alsaleh G, Pichot A, Ostermann E, Zuber G, Frisch B, et al. MiR-20a regulates ASK1 expression and TLR4-dependent cytokine release in rheumatoid fibroblast-like synoviocytes. Ann Rheum Dis 2013;72:1071-9.

14. Philippe L, Alsaleh G, Bahram S, Pfeffer S, Georgel P. The miR-17 92 cluster: a key player in the control of inflammation during rheumatoid arthritis. Front Immunol 2013;4:70.

15. Hill DA, Ivanovich J, Priest JR, Gurnett CA, Dehner LP, Desruisseau D, et al. DICER1 mutations in familial pleuropulmonary blastoma. Science 2009;325:965.

16. Bahubeshi A, Tischkowitz M, Foulkes WD. miRNA processing and human cancer: DICER1 cuts the mustard. Sci Transl Med 2011;3:111ps46.

17. Heravi-Moussavi A, Anglesio MS, Cheng SW, Senz J, Yang W, Prentice L, et al. Recurrent somatic DICER1 mutations in nonepithelial ovarian cancers. N Engl J Med 2012;366:234-42.

18. Rio Frio T, Bahubeshi A, Kanellopoulou C, Hamel N, Niedziela M, Sabbaghian N, et al. DICER1 mutations in familial multinodular goiter with and without ovarian Sertoli-Leydig cell tumors. JAMA 2011;305:68-77.

19. Darrat I, Bedoyan JK, Chen M, Schuette JL, Lesperance MM Novel DICER1 mutation as cause of multinodular goiter in children. Head Neck 2013;35:E369-71.

20. Wu MK, Sabbaghian N, Xu B, Addidou-Kalucki S, Bernard C, Zou D, et al. Biallelic DICER1 mutations occur in Wilms tumours. J Pathol 2013;230:154-64.

21. Choong CS, Priest JR, Foulkes WD. Exploring the endocrine manifestations of DICER1 mutations. Trends Mol Med 2012;18: 503-5.

22. Melo SA, Esteller M. Disruption of microRNA nuclear transport in human cancer. Semin Cancer Biol 2014;27:46-51.

23. Aletaha D, Neogi T, Silman AJ, Funovits J, Felson DT, Bingham CO III, et al. 2010 rheumatoid arthritis classification criteria: an American College of Rheumatology/European League Against Rheumatism collaborative initiative. Arthritis Rheum 2010;62:2569-81.

24. Neff L, Zeisel M, Sibilia J, Scholler-Guinard M, Klein JP, Wachsmann D. NF- $\kappa$ B and the MAP kinases/AP-1 pathways are both involved in interleukin-6 and interleukin-8 expression in fibroblast-like synoviocytes stimulated by protein I/II, a modulin from oral streptococci. Cell Microbiol 2001;3:703-12.

25. Otsuka M, Jing Q, Georgel P, New L, Chen J, Mols J, et al. Hypersusceptibility to vesicular stomatitis virus infection in Dicer1-deficient mice is due to impaired miR24 and miR93 expression. Immunity 2007;27:123-34.

26. Ostermann E, Tuddenham L, Macquin C, Alsaleh G, SchreiberBecker J, Tanguy M, et al. Deregulation of type I IFNdependent genes correlates with increased susceptibility to cytomegalovirus acute infection of dicer mutant mice. PloS One 2012; 7:e43744

27. Ditzel HJ. The $\mathrm{K} / \mathrm{BxN}$ mouse: a model of human inflammatory arthritis. Trends Mol Med 2004;10:40-5.

28. Ji H, Pettit A, Ohmura K, Ortiz-Lopez A, Duchatelle V, Degott $\mathrm{C}$, et al. Critical roles for interleukin 1 and tumor necrosis factor $\alpha$ in antibody-induced arthritis. The J Exp Med 2002;196:77-85.

29. Wiesen JL, Tomasi TB. Dicer is regulated by cellular stresses and interferons. Mol Immunol 2009;46:1222-8.

30. Eyre S, Bowes J, Diogo D, Lee A, Barton A, Martin P, et al. High-density genetic mapping identifies new susceptibility loci for rheumatoid arthritis. Nat Genet 2012;44:1336-40.

31. Diogo D, Okada Y, Plenge RM. Genome-wide association studies to advance our understanding of critical cell types and pathways in rheumatoid arthritis: recent findings and challenges. Curr Opin Rheumatol 2014;26:85-92.

32. Ammari M, Jorgensen C, Apparailly F. Impact of microRNAs on the understanding and treatment of rheumatoid arthritis. Curr Opin Rheumatol 2013;25:225-33.

33. Yang B, Chen J, Li Y, Zhang J, Li D, Huang Z, et al. Association of polymorphisms in pre-miRNA with inflammatory biomarkers in rheumatoid arthritis in the Chinese Han population. Hum Immunol 2012;73:101-6.

34. Hashemi M, Eskandari-Nasab E, Zakeri Z, Atabaki M, Bahari G, Jahantigh M, et al. Association of pre-miRNA-146a rs2910164 and premiRNA-499 rs3746444 polymorphisms and susceptibility to rheumatoid arthritis. Mol Med Rep 2013;7:287-91.

35. Charles JF, Hsu LY, Niemi EC, Weiss A, Aliprantis AO, Nakamura MC. Inflammatory arthritis increases mouse osteoclast precursors with myeloid suppressor function. J Clinical Invest 2012; 122:4592-605.

36. Kleyer A, Schett G. Arthritis and bone loss: a hen and egg story. Curr Opin Rheumatol 2014;26:80-4.

37. Kurzynska-Kokorniak A, Koralewska N, Pokornowska M, Urbanowicz A, Tworak A, Mickiewicz A, et al. The many faces of Dicer: the complexity of the mechanisms regulating Dicer gene expression and enzyme activities. Nucleic Acids Res 2015;43:4365-80.

38. Kaneko H, Dridi S, Tarallo V, Gelfand BD, Fowler BJ, Cho WG, et al. DICER1 deficit induces Alu RNA toxicity in agerelated macular degeneration. Nature 2011;471:325-30.

39. Tarallo V, Hirano Y, Gelfand BD, Dridi S, Kerur N, Kim Y, et al. DICER1 loss and Alu RNA induce age-related macular degeneration via the NLRP3 inflammasome and MyD88. Cell 2012;149:847-59.

40. Emde A, Hornstein E. miRNAs at the interface of cellular stress and disease. EMBO J 2014;33:1428-37.

41. Dias C, Isenberg DA. Susceptibility of patients with rheumatic diseases to B-cell non-Hodgkin lymphoma. Nat Rev Rheumatol 2011;7:360-8.

42. Franks AL, Slansky JE. Multiple associations between a broad spectrum of autoimmune diseases, chronic inflammatory diseases and cancer. Anticancer Res 2012;32:1119-36.

43. Pap T, Aupperle KR, Gay S, Firestein GS, Gay RE. Invasiveness of synovial fibroblasts is regulated by p53 in the SCID mouse in vivo model of cartilage invasion. Arthritis Rheum 2001;44:676-81. 